\title{
Computational classification of animals for a highway detection system
}

\section{Classificação computacional de animais para sistema de detecção nas rodovias}

\author{
Denis Sato ${ }^{1}$ (D); Adroaldo José Zanella ${ }^{2}$; Ernane Xavier Costa ${ }^{3}$ \\ ${ }^{1}$ Universidade de São Paulo, Faculdade de Medicina Veterinária e Zootecnia, Pirassununga - SP, Brasil \\ ${ }^{2}$ Universidade de São Paulo, Faculdade de Medicina Veterinária e Zootecnia, Departamento de Medicina Veterinária Preventiva e Saúde \\ Animal, Pirassununga - SP, Brasil \\ ${ }^{3}$ Universidade de São Paulo, Faculdade de Zootecnia e Engenharia de Alimentos, Departamento de Ciências Básicas, Pirassununga - SP, \\ Brasil
}

\begin{abstract}
Vehicle-animal collisions represent a serious problem in roadway infrastructure. To avoid these roadway collisions, different mitigation systems have been applied in various regions of the world. In this article, a system for detecting animals on highways is presented using computer vision and machine learning algorithms. The models were trained to classify two groups of animals: capybaras and donkeys. Two variants of the convolutional neural network called Yolo (You only look once) were used, Yolov4 and Yolov4-tiny (a lighter version of the network). The training was carried out using pre-trained models. Detection tests were performed on 147 images. The accuracy results obtained were 84.87\% and $79.87 \%$ for Yolov4 and Yolov4-tiny, respectively. The proposed system has the potential to improve road safety by reducing or preventing accidents with animals.
\end{abstract}

Keywords: Machine-learning. Vehicle-animal collisions. Computational vision.

\section{RESUMO}

As colisões entre veículos e animais representam um sério problema na infraestrutura rodoviária. Para evitar tais acidentes, medidas mitigatórias têm sido aplicadas em diferentes regiões do mundo. Neste projeto é apresentado um sistema de detecção de animais em rodovias utilizando visão computacional e algoritmo de aprendizado de máquina. Os modelos foram treinados para classificar dois grupos de animais: capivaras e equídeos. Foram utilizadas duas variantes da rede neural convolucional chamada Yolo (você só vê uma vez) - Yolov4 e Yolov4-tiny (versão mais leve da rede) - e o treinamento foi realizado a partir de modelos pré-treinados. Testes de detecção foram realizados em 147 imagens e os resultados de precisão obtidos foram de 84,87\% e 79,87\% para Yolov4 e Yolov4-tiny, respectivamente. O sistema proposto tem o potencial de melhorar a segurança rodoviária reduzindo ou prevenindo acidentes com animais.

Palavras-chave: Aprendizado de máquina. Acidentes rodoviários com animais. Visão computacional.

Correspondence to:

Denis Sato

Universidade de São Paulo, Faculdade de Medicina Veterinária

e Zootecnia, Departamento de Medicina Veterinária

Preventiva e Saúde Animal, Campus Fernando Costa

Av. Duque de Caxias Norte, 225, Jardim Elite

CEP: 13635-900, Pirassununga - SP, Brasil

e-mail: denissato@usp.br

Received: September 21, 2020

Approved: March 15, 2021
How to cite: Sato D, Zanella AJ, Costa EX. Computational classification of animals for a highway detection system. Braz J Vet Res Anim Sci. 2021;58(special issue):e174951. https://doi.org/10.11606/issn.1678-4456.bjvras.2021.174951

\section{Introduction}

Several studies have shown that vehicle-animal collisions are a global problem. The most serious accidents involve larger body size animals of both native and naturalized species, such as wild and feral swine and different deer species in 
Europe and the United States (Hothorn et al., 2015; Pynn \& Pynn, 2004; Rodríguez-Morales et al., 2013; Sullivan, 2011), wolves in eastern Canada (Lesmerises et al., 2013), elephants in Malaysia (Wadey et al., 2018), camels in Arab countries (Al-Ghamdi \& AlGadhi, 2004; Al-Sebai \& Al-Zahrani, 1997), and donkeys in the Northeast Brazil ${ }^{1}$.

These accidents are responsible for the injuries and deaths of people and animals every year. Also, the monetary losses amount to billions of dollars (Bruinderink \& Hazebroek, 1996; Huijser et al., 2013; Olson et al., 2014; Sullivan, 2011). A previous study established that from 2010 to 2016, 25,075 accidents involving fauna occurred on Brazilian federal highways, resulting in 613 recorded human deaths, 2,933 serious injuries, and 7,559 minor injuries (Tavares et al., 2017).

To reduce accidents, several mitigating actions have been proposed. The infrastructure of underpasses and overpasses (Dodd et al., 2004; Tissier et al., 2016), electronic devices with presence sensors (Biblioteca Virtual FAPESP, 2020; Grace et al., 2017), and lighting and odorous devices (Bíl et al., 2018; D’Angelo \& van der Ree, 2015; Kušta et al., 2015) are some examples.

Computational vision systems for animal detection have been applied to different purposes (Christiansen et al., 2014; Rangda \& Hanchate, 2014; Sharma \& Shah, 2017). Concerning transportation activities, computational vision animal detection systems have been developed in cars (Agrawal \& Singh, 2020; Consumer Reports, 2020) and railway networks (Ye et al., 2018; Yu et al., 2018). Because of the evolution of computational power and convolutional neural networks, the algorithms are now capable of realizing real-time object detection with the use of video cameras. For example, Japan is using around 13,000 video cameras and artificial intelligence to monitor highways to detect traffic problems and increase safety (Fujitsu Blog, 2019).

A video is a moving picture sequence defined by its temporal resolution in frames per second (fps). Therefore, the object detection algorithms used for this purpose must be fast to compute many detections per second. In recent years, the development of object detection frameworks like Yolo (Bochkovskiy et al., 2020), Mask R-CNN (He et al., 2017), and Single Shot Multibox Detector (Liu et al., 2016), has made real-time detection possible. Also, using infrared thermal cameras makes it possible to detect the animals at night or in low visibility weather conditions (e.g., fog, rain, snowfall) (Christiansen et al., 2014; Perissinotto et al.,

Gameiro MBP, Clancy C, Zanella AJ. Between freedom and abandonment: social representations of free-roaming donkeys in the Brazilian Northeast. Anthrozoos. 2020. [under evaluation].
2009; Oishi et al., 2018; Zhou, 2013). Here we describe a system developed for detecting the presence of animals on highways using computational vision and machine learning in an embedded system.

\section{Materials and Methods}

\section{Model training}

For model training, 2,000 images of capybaras and equines (donkeys and horses) were downloaded from Google Images, 1,000 for each group, using a batch download extension (Chrome Web Store, 2020). The objects of interest (animals) in each image were labeled with bounding boxes using the LabelImg software (Tzutalin, 2015) given the capivara class name for capybaras, and equidae class name for equines. The labeling process for object detection consists of delimiting the pixels that represent the object of interest in the image and assigning it a class name. After drawing a bounding box around the object, the label software writes the object's pixel coordinates in an external file. Figure 1 provides a visualization of the labeling process and coordinates file content generated.

For example, the center frame provides a visualization of an $x m l$ (Extensible Markup Language) file content and the right frame a $t x t$ (text) file content. The content of the files are the drawn bounding box coordinates.

The dataset was divided into two groups: training $(n=1800)$ and validation $(\mathrm{n}=200)$. The Yolov4 (Bochkovskiy et al., 2020), an artificial convolutional neural network (Convnet), was selected for the classification training. The framework used to set up the training was the Darknet (Bochkovskiy, c2020). Two different training sessions were carried out, the full version of Yolov4 and a lighter version called Yolov4-tiny, using pre-trained models (Bochkovskiy, 2020). The Yolov4 was trained in cloud computing ambient (Google, 2020) (GPU Nvidia Tesla k80 24GB) and Yolov4-tiny in a desktop computer (AMD Ryzen 2700x, 16GB RAM, GPU Nvidia GTX 1050ti $4 \mathrm{~GB}$ ), up to 8,000 iterations for each model.

\section{Performance evaluation}

After training, 147 images were used to test the detector performance. These images were not used in the training and were labeled using the LabelImg software (Tzutalin, 2015). This labeling is realized to generate the groundtruth files that have the coordinates (bounding boxes) about object localization in each picture. The detection results (bounding boxes) are then compared with the information for each object. The comparative results between detections and ground-truth coordinates were computed 


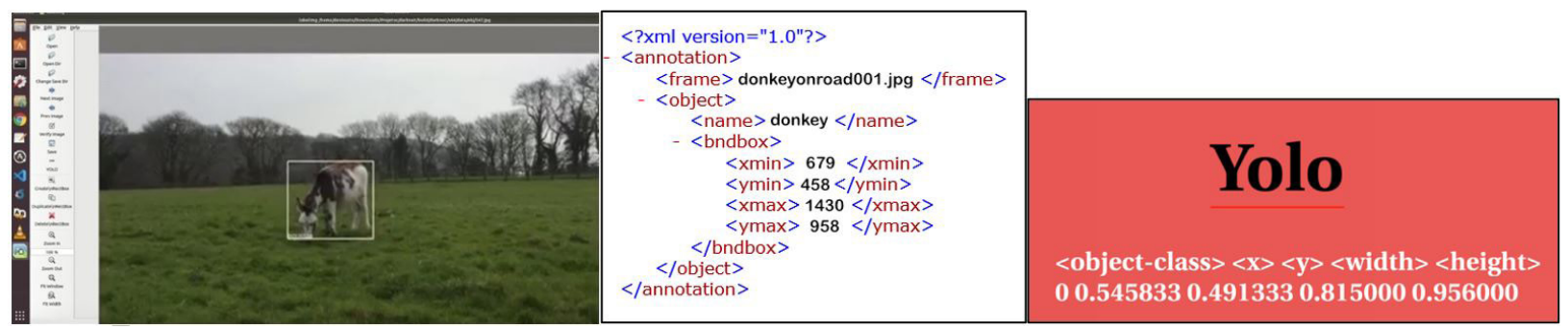

Figure 1 - Labeling the object of interest in an image. After drawing a bounding box around the object of interest (left frame), the label software generates a file with its coordinates. The format of the file depends on the chosen algorithm requirements.

with the software mAP (Cartucho, 2015; Cartucho et al., 2018) and were performed according to the Pascal VOC Challenge 2012 metrics modified (Everingham \& Winn, 2012). In addition to the precision results graphics, the mAP software records comparative bounding boxes in each image for visual analysis. We configured the mAP software to present green bounding boxes as true positive detections, red as false positives, and yellow for undetected objects (false negatives). The ground-truth bounding boxes are presented in blue.

The results of the detection are classified by decreasing confidence level and compared to the ground-truth data. The detection result is considered positive when the value of Intersection over Union is greater or equal to 0.1 ( $\mathrm{IoU} \geq$ $10 \%)$. Multiple detections for the same object are counted as false positives. The IoU is calculated by the Equation 1:

$$
I o U=\frac{\operatorname{area}\left(B_{p} \cap B_{g t}\right)}{\operatorname{area}\left(B_{p} \cup B_{g t}\right)} \geq 0,1
$$

where $B_{p}$ is the predicted bounding box area and $B_{\mathrm{gt}}$ the ground-truth. Figure 2 provides a visual explanation of the IoU.

From the results of IoU, a precision/recall curve with precision monotonically decreased by setting the precision for recall $r$ to the maximum precision obtained for any recall $r^{\prime}>r$ is computed. The average precision (AP) for each class is calculated as the area under this curve by numerical integration. No approximation is involved since the curve is piecewise constant. The precision of all classes is presented as the mean average precision ( $\mathrm{mAP}$ ) calculated by the mean of all AP's.

The accuracy (Ac) of the models was calculated by the Equation 2:

$$
A c=\frac{T P_{\text {det }}}{G t+F P_{\text {det }}}
$$

where $T P_{\text {det }}$ is the total number of true positives detected, $G t$ is the total number of objects in the ground-truth data, and $F P_{d e t}$ is the total number of false positives detected. The speed test was performed with recorded videos were carried

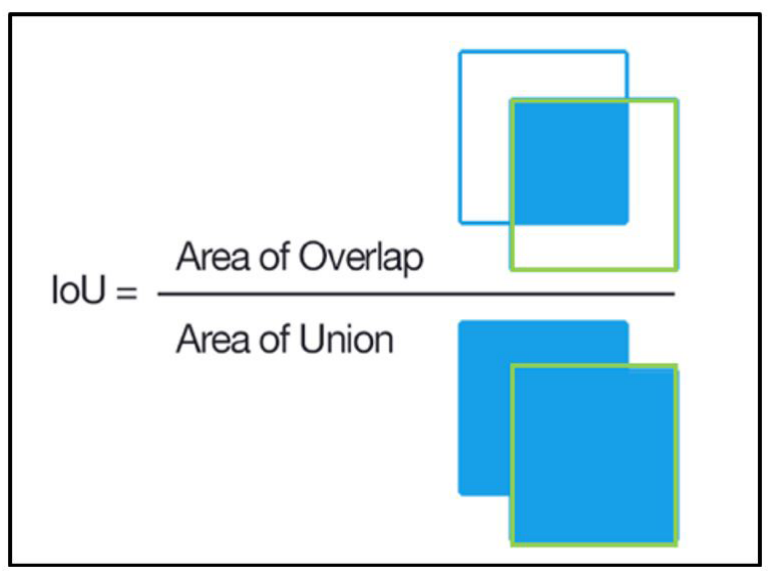

Figure 2 - Intersect over Union. Intersection over Union is an evaluation metric used to measure the accuracy of an object detector on a particular dataset. In the numerator, the area of overlap between the predicted bounding box (green) and the ground-truth bounding box (blue) is computed. The denominator is the area of union, which is the sum of the area of both bounding boxes.

out for capybaras and donkeys in a desktop computer with GPU GTX 1050Ti and are presented in frames per second.

\section{Embedded system}

The embedded system consists of a video camera, an Nvidia Jetson TX2 processing unit, a traffic signboard with an adapted light signal, and a solar panel power supply. For the luminous alert function, a modification was made in the darknet_video.py algorithm (Bochkovskiy, 2020) using PyFirmata library (Bruijn, 2020), to establish communication with an Arduino 2560 mega-development board. During tests on video files, the operation of the light alert was observed experimentally using light-emitting diodes (LED). Figure 3 shows the assembly scheme and Figure 4 a virtual view of the proposed system.

\section{Results and Discussion}

The comparative detection results are presented in Figure 5.

Considering both models, the capybara class presented a higher number of false positives $(n=49)$ compared to the 
equidae class ( $\mathrm{n}=31$ ). This may be related to the greater number of individuals in the group and which may not have been labeled in the ground-truth data but were detected during the tests. Undetected objects are the main problem for an alert system for the presence of animals on the highways. In this case, the equidae class presented a higher number of undetected objects $(n=40)$ compared to the capybara class $(n=7)$. Nevertheless, analyzing the detections frame by frame, in most images that have undetected objects (false-

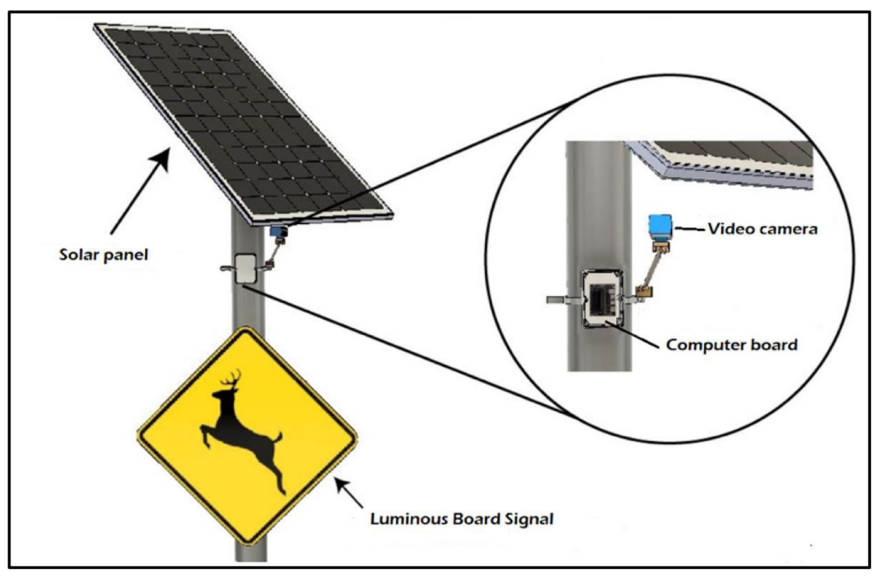

Figure 3-Assembly scheme of the embedded system. negatives - FNs), there are also TP detections. Thus, from the point of view of alerting to the presence of animals on the highway, such occurrences in real-time would not result in a problem of the absence of an alert if there is at least a positive detection in the scene. Also, it is possible to verify that the majority of false-positive (FP) detections occurred in frames with true-positive (TP) detections. Two examples of these occurrences are presented in Figure 6.

Different challenges, such as occlusion, varied lighting conditions, spatial position, and scale, must be handled by the algorithm during object detection. False positives commonly occur due to localization error, confusion with the background or similar objects, and an insufficient amount of overlap (IoU). A false negative can be the result of low confidence attributed to the object, occlusions, truncation, small size, or unusual point of view (Ponce et al., 2006; Hoiem et al., 2012; Pathak et al., 2018).

The metric used in object detection challenges to assess model performance is based on the localization of the object in the image according to the IoU threshold (Common Objects in Context, 2020; PASCAL VOC Project, 2012; Russakovsky et al., 2015). Furthermore, we decided

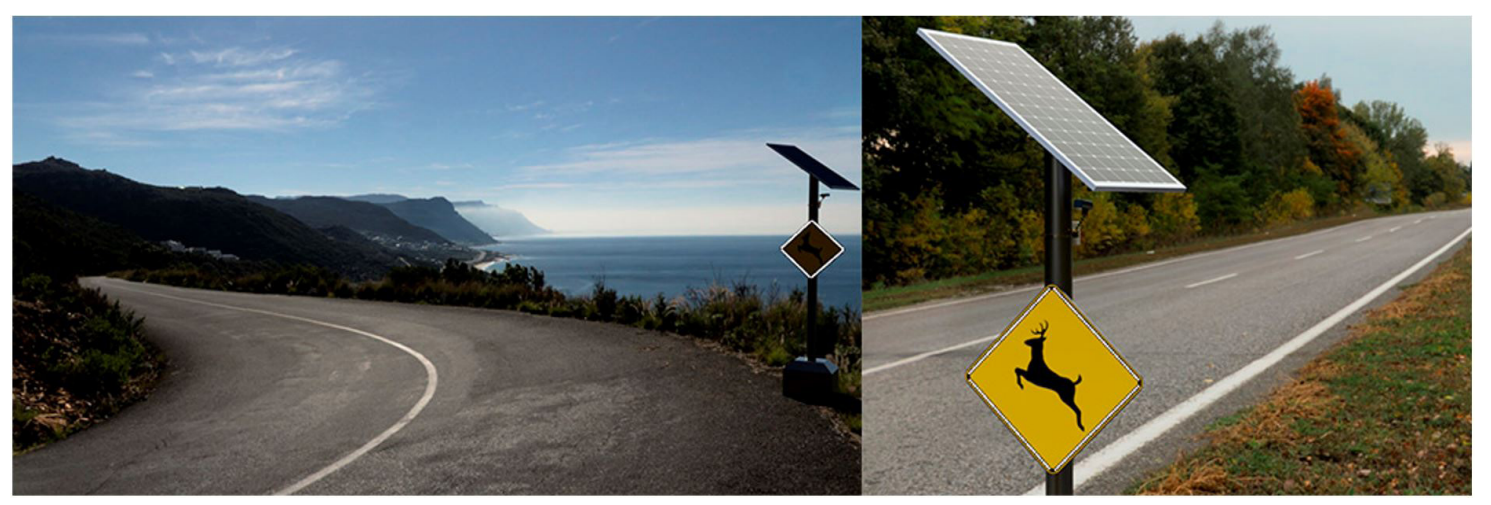

Figure 4 - Virtualization of the final signboard light alert system. Generated with Autodesk ${ }^{\circledR}$ Fusion 360 Educational license.

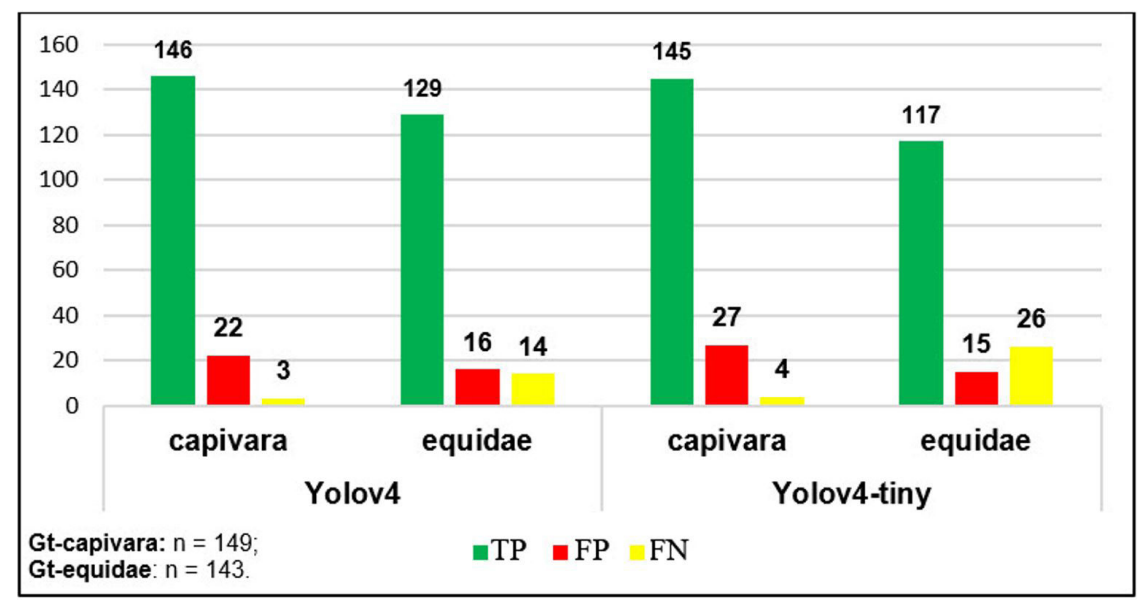

Figure 5 - Comparative detection results between Yolov4 and Yolov4-tiny. Source: author. TP - true-positives; FP - false-positives; FN - false-negatives; Gt-capivara - number of objects in ground-truth. Gt-equidae - number of objects in ground-truth. Data computed in mAP (IoU $\geq 0.1)$ (Cartucho, 2015). 


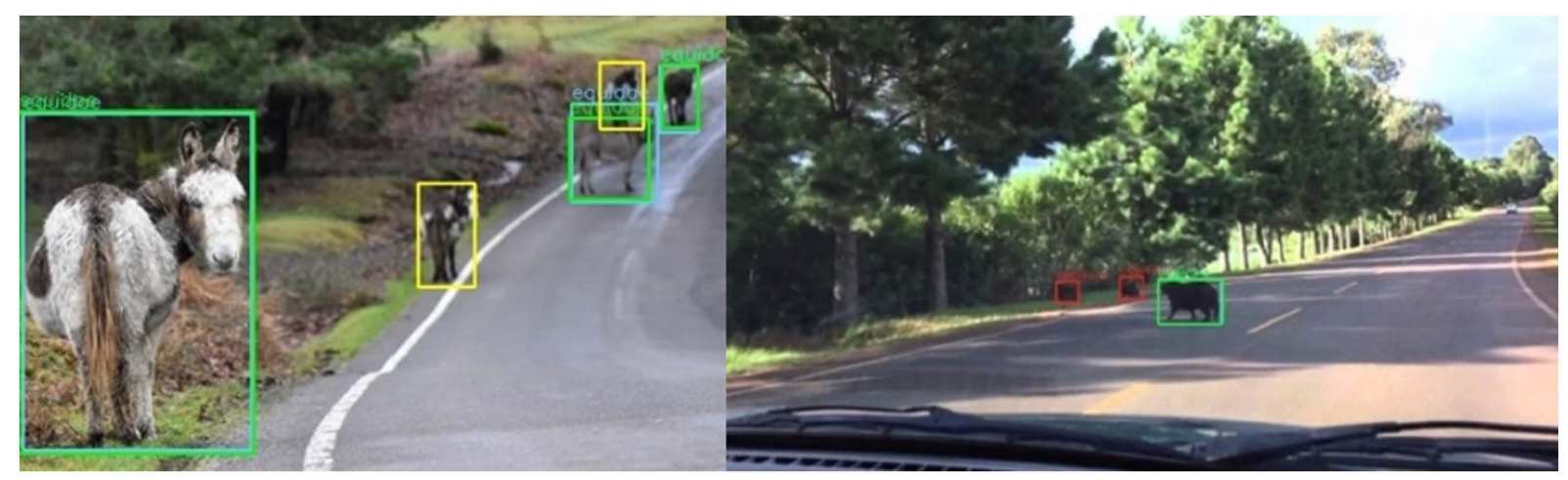

Figure 6 - Examples of false-positive and undetected objects. Source: author. Bounding-boxes: green - true-positive; red - falsepositive; yellow - false-negative. Generated with mAP (Cartucho, 2015).

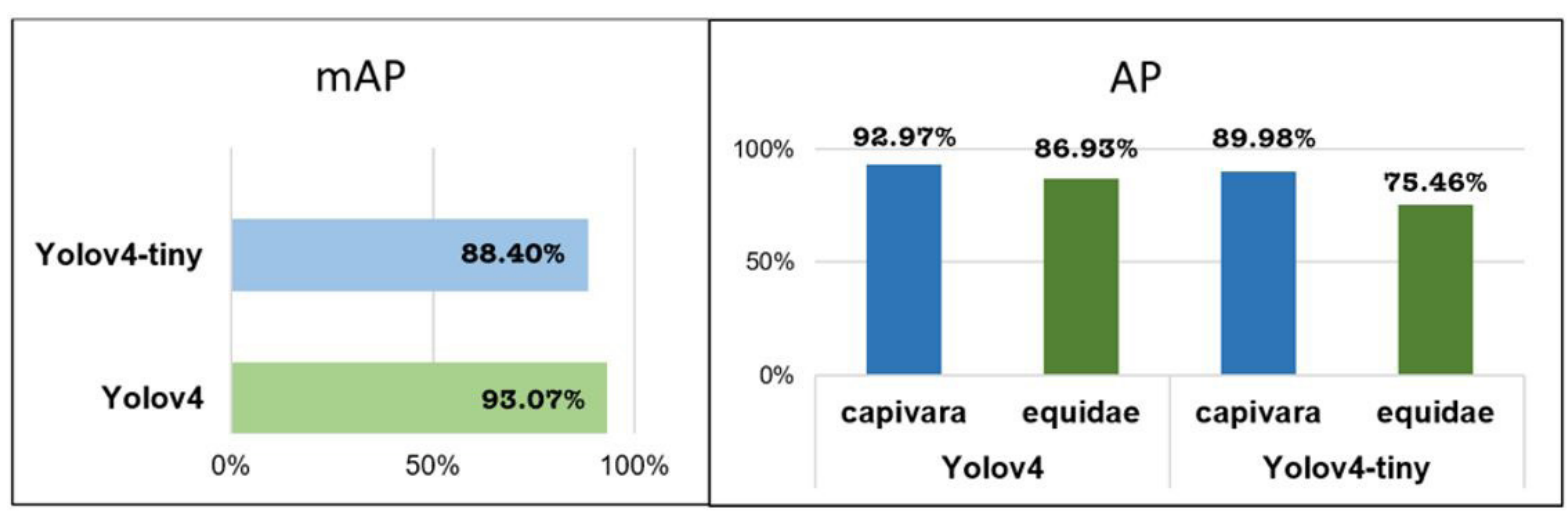

Figure 7 - Comparative precision results of Yolov4 and Yolov4-tiny. Source: author. $\mathbf{m A P}$ - mean average precision for all classes; AP - average precision of each class. Data computed in $\mathrm{mAP}$ ( $\mathrm{IoU} \geq 0.1)$ (Cartucho, 2015).

to use a lower overlap value ( $\mathrm{IoU} \geq 0.1$ ) in the detection evaluations instead of the one recommended in the Pascal VOC Challenge 2012 ( $\mathrm{IoU} \geq 0.5$ ) to increase sensitivity. Thus, true-positive detections are computed even when only part of the animal's body is detected. Subsequently, the system performance evaluation metric must be modified to consider as true-positive the entire frame if any object of interest is detected.

Detection errors can be minimized by adjusting the training hyper-parameters (anchoring, resolution, optimizers), normalizing the dataset, using negative samples, and increasing the volume of samples by class (Chu et al., 2016; Alshalali \& Josyula, 2018). Also, the Yolov4 presents a list of these adjustments that can be set up to improve the detection accuracy of custom models (Bochkovskiy, c2020).

Both models showed a similar precision performance, with a slight advantage for the full version of Yolov4. The results of average precision by class (AP) and the average detector precision (mAP) of each model are presented in Figure 7.

Considering the adopted performance evaluation metric, the precision results presented for both classes, in both models, were satisfactory. The accuracy results were $84.87 \%$ and $79.87 \%$ for Yolov4 and Yolov4-tiny, respectively. Analyzing the dataset used for training, although the same number of images were selected for each class, a greater number of objects of the capivara class $(\mathrm{n}=2412)$ were labeled than the objects of the equidae class ( $\mathrm{n}=1541$ ). This explains the differences in detection results between the classes. One of the most important steps for image classification is the management of data for learning (Munappy et al., 2019). During tests on a highway, it will be possible to increase the dataset by obtaining new images and promote reinforced learning. Besides, other species of animals could be added to training according to local necessity.

Although there are many datasets available for download on the internet (Abu-El-Haija et al., 2016; Fei-Fei et al., 2010; Huang et al., 2007; Khosla et al., 2011; Lin et al., 2014), finding a large number of images for specific classes is a significant challenge. The models were trained using pre-trained weights, a technique called transfer learning. During training, the learned data is stored in files called weights. Transfer learning is the use of information from one domain and transferring it to another related domain. This technique is used when dataset building can be difficult or expensive (Weiss et al., 2016; Zhuang et al., 2019). Although it has been demonstrated that predictive results can be obtained with high precision in an image classification model without the use of pre-trained weights (He et al., 2019), a 


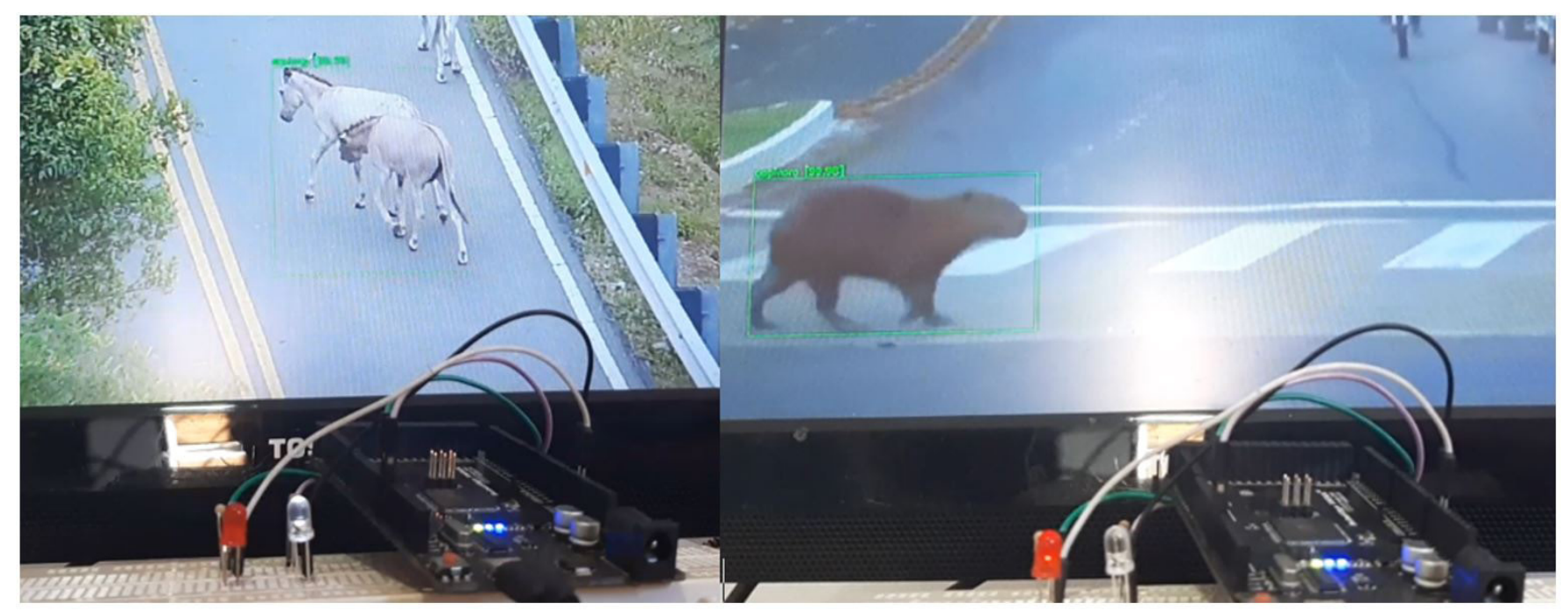

Figure 8 - Light alert for true-positive detections. Left frame - white LED blinks for equidae class detection; Right frame - red LED blinks for capivara class detection.

large volume of samples was used for training and validation (35,000 images for training and 5,000 images for validation). When a test was performed with many images similar to this work, there was an excess of adjustment in the model due to the limited number of samples, reducing the average precision value in the predictions $(\mathrm{AP}=9,9$ - pre-trained model; $\mathrm{AP}=3.5$ model without pre-trained weights). Also, the transfer learning required less lead time to converge the weights to an asymptotic point, and a considerably smaller number of samples can be used for training (Chollet, 2016; Rawat \& Wang, 2017).

The speed results were $40 \mathrm{fps}$ using Yolo4-tiny and $28 \mathrm{fps}$ using Yolov4. During the video tests, the luminous alert could be verified by a white LED (Light Emitting Diode) for equidae class and a red LED for capivara class as presented in Figure 8. The different colors of the light alert have been set up only for test purposes. The configuration will be modified for the embedded system and new experiments will be carried out.

\section{Conclusions}

The models presented high precision in detecting the objects tested. Despite both classes having high levels of a true positive detection, the equidae class presented a greater number of undetected objects (false negatives) compared with the capivara class. The issue related to the undetected objects is the most important one to consider in a highway collision avoidance system and needs to be adjusted accordingly. The precision results for the equidae class were lower than those of the capybara class because of the smaller number of samples that were used for classification in training. However, tests were performed on still images and video files. It is necessary to make corrections in the dataset for new training and perform experiments in real-time situations.

In the future, other animal species will be included, and real-time tests in various light and visibility levels will be carried out on the experimental roadway located near the Department of Preventive Veterinary Medicine and Animal Health (University of São Paulo - Pirassununga/SP). The length of the experimental road is 300 meters, and the detection system will be evaluated for donkeys, capybaras, dogs, bovines, and horses positioned at 100, 200, and 300 meters.

The system proposed in this work has the potential to contribute to improving animal welfare and road safety due to accidents involving animals. The next steps in the project will clarify whether the proposals discussed can be confirmed or rejected.

\section{Conflict of Interest}

The authors whose names are listed in this publication certify that they have NO affiliations with or involvement in any organization or entity with any financial interest or non-financial interest in the subject matter or materials discussed in this manuscript.

\section{Ethics Statement}

This project was reviewed and approved by the Ethical Committee of the School of Veterinary Medicine and Animal Science under number CEUA No 4526170418.

\section{Acknowledgements}

Special thanks to The Donkey Sanctuary, Arteris GroupIntervias, Veterinary Medicine Foundation (FUMVET), and the University of São Paulo for supporting this research. 


\section{References}

Abu-El-Haija S, Kothari N, Lee J, Natsev P, Toderici G, Varadarajan B, Vijayanarasimhan S. YouTube-8M: a largescale video classification benchmark 2016 Sept 17 [cited 2020 Sept 21]. In: arXiv [Internet]. New York: Cornell University; [2020]. Available from: http://arxiv.org/abs/1609.08675.

Agrawal R, Singh N. Lane detection and collision prevention system for automated vehicles. In: Iyer B, Rajurkar AM, Gudivada V, editors. Applied computer vision and image processing [Internet]. Singapore: Springer; 2020 [cited 2021 May 7]. p. 46-59. (Advances in Intelligent Systems and Computing). Available from: http://dx.doi.org/10.1007/978981-15-4029-5_5.

Al-Ghamdi AS, AlGadhi SA. Warning signs as countermeasures to camel-vehicle collisions in Saudi Arabia. Accid Anal Prev. 2004;36(5):749-60. http://dx.doi.org/10.1016/j. aap.2003.05.006.

Al-Sebai MW, Al-Zahrani S. Cervical spinal injuries caused by collision of cars with camels. Injury. 1997;28(3):191-4. http://dx.doi.org/10.1016/S0020-1383(96)00191-X.

Alshalali T, Josyula D. Fine-tuning of pre-trained deep learning models with extreme learning machine [Internet]. Proceedings - 2018 International Conference on Computational Science and Computational Intelligence; 2018 Dec 12-14; Las Vegas, Nevada [Internet]. New York: IEEE; 2018 [cited 2021 May7]. p. 469-473. Available from: https://ieeexplore. ieee.org/document/8947855. http://dx.doi.org/10.1109/ CSCI46756.2018.00096.

Biblioteca Virtual FAPESP [Internet]. São Paulo: FAPESP; 2020. Desenvolvimento de sistema de detecção animal antiatropelamento em rodovias - "Passa Bicho" [cited 2020 Sept 21]. Available from: http://www.bv.fapesp.br/pt/ auxilios/93334/desenvolvimento-de-sistema-de-deteccaoanimal-antiatropelamento-em-rodovias-passa-bicho/.

Bíl M, Andrášik R, Bartonička T, Křivánková Z, Sedoník J. An evaluation of odor repellent effectiveness in prevention of wildlife-vehicle collisions. J Environ Manage. 2018;205:209-14. Available from: https://ieeexplore.ieee.org/document/8947855. http://dx.doi.org/10.1016/j.jenvman.2017.09.081.

Bochkovskiy A. Darknet - Yolo-V4 and Yolo-v3/v2 for Windows and Linux. [Date unknown] - [cited $2020 \mathrm{Sept}$ 21]. In: GitHub [Internet]. [place unknown]; c2020. [cited 2020 Sept 21]. Available from: https://github.com/ AlexeyAB/darknet.
Bochkovskiy A, Wang C-Y, Liao H-YM. YOLOv4: optimal speed and accuracy of object detection. 2020 Apr 23 [cited 2020 Sept 21]. In: arXiv [Internet]. New York: Cornell University; 2020. Available from: http://arxiv.org/ abs/2004.10934.

Bruijn T. PyFirmata [2020 Jun 23] - [cited 2020 Sept 21]. In: GitHub [Internet]. [place unknown]; c2020. Available from: https://github.com/tino/pyFirmata.

Bruinderink GWTAG, Hazebroek E. Ungulate traffic collisions in Europe. Conserv Biol. 1996;10(4):1059-67. http://dx.doi.org/10.1046/j.1523-1739.1996.10041059.x.

Cartucho J, Ventura R, Veloso M. Robust object recognition through symbiotic deep learning in mobile robots. In: 2018 IEEE/RSJ International Conference on Intelligent Robots and Systems (IROS); 2018; Madrid, Spain [Internet]. New York: IEEE; 2018 [cited 2020 Sept 21]. p.2336-41. http:// dx.doi.org/10.1109/IROS.2018.8594067.

Cartucho J. mAP. 2005 May 29 [cited 2020 Sept 21]. In: GitHub [Internet]. [place unknown]; c2020. Available from: https:/github.com/Cartucho/mAP.

Chollet F. Building powerful image classification models using very little data. 2016 Jun 5 [cited 2020 May 20]. In: The Keras Blog [Internet]. [place unknown]; 2020. Available from: https://blog.keras.io/building-powerfulimage-classification-models-using-very-little-data.html.

Christiansen P, Steen KA, Jørgensen RN, Karstoft H. Automated detection and recognition of wildlife using thermal cameras. Sensors. 2014;14(8):13778-93. http:// dx.doi.org/10.3390/s140813778.

Chrome Web Store [Internet]. Fatkun Batch Download Image - Chrome extension. [place unknown]; 2020 [cited 2020 Sept 21]. Available from: https://chrome.google. com/webstore/detail/fatkun-batch-download-ima/ nnjjahlikiabnchcpehcpkdeckfgnohf.

Chu B, Madhavan V, Beijbom O, Hoffman J, Darrell T. Best practices for fine-tuning visual classifiers to new domains. In: Hua G, Jégou, H, editors. Computer Vision - ECCV 2016 Workshops. Switzerland: Springer; 2016. p. 435-42. (Lecture Notes in Computer Science, including subseries Lecture Notes in Artificial Intelligence and Lecture Notes in Bioinformatics; v. 9915). 
Common Objects in Context - COCO. COCO 2020 Object Detection Task [Internet]. [place unknown]; 2020 [cited 2020 Sept 21]. Available from: https://cocodataset. org/\#detection-2020.

Consumer Reports [Internet]. Yonkers, NY: CR; c2020. Cars with advanced safety systems [cited 2020 Sept 21]. Available from: https://www.consumerreports.org/carsafety/cars-with-advanced-safety-systems/.

D’Angelo G, van der Ree R. Use of reflectors and auditory deterrents to prevent wildlife-vehicle collisions. In: van der Ree R, Smith DJ, Grilo C, editors. Handbook of road ecology [Internet]. Hoboken: John Wiley \& Sons; 2015 [cited 2021 May 7]. p. 213-8. Available from: https://onlinelibrary.wiley. com/doi/abs/10.1002/9781118568170.ch25. http://dx.doi. org/10.1002/9781118568170.ch25.

Dodd CK Jr, Barichivich WJ, Smith LL. Effectiveness of a barrier wall and culverts in reducing wildlife mortality on a heavily traveled highway in Florida. Biol Conserv. 2004;118(5):61931. http://dx.doi.org/10.1016/j.biocon.2003.10.011.

Everingham M, Winn J. The PASCAL Visual Object Classes Challenge 2012 (VOC2012) development kit [Internet]. [place unknown]: PASCAL VOC; 2012 May 18 [cited 2020 Sept 21]. Available from: http://host.robots.ox.ac.uk/pascal/ VOC/voc2012/devkit_doc.pdf.

Fei-Fei L, Deng J, Li K. ImageNet: constructing a largescale image database. J Vis. 2010;9(8):1037. http://dx.doi. org/10.1167/9.8.1037.

Fijitsu Blog [Internet]. [place unknown]: Fujitsu; 2019. How Fujitsu is using AI to make Japan's roads and rivers safe [cited 2020 Sept 21]. Available from: https://blog.global. fujitsu.com/fgb/2019-11-20/how-fujitsu-is-using-ai-tomake-japans-roads-and-rivers-safer/\#: :text=Today\%2C in order to monitor,national highways across the country.

Google. Colaboratory [Internet]. [place unknown]; 2020 [cited 2020 Feb 12]. Available from: https://colab.research. google.com/.

Grace MK, Smith DJ, Noss RF. Reducing the threat of wildlife-vehicle collisions during peak tourism periods using a Roadside Animal Detection System. Accid Anal Prev. 2017;109:55-61. http://dx.doi.org/10.1016/j.aap.2017.10.003.

He K, Gkioxari G, Dollar P, Girshick R. Mask R-CNN. In: Proceedings of the IEEE International Conference on Computer Vision; 2017; Venice, Italy [Internet]. New York: IEEE; 2017 [2021 May 7]. p. 2980-8. Available from: https://ieeexplore.ieee.org/document/8237584. https://doi. org/10.1109/ICCV.2017.322.

He K, Girshick R, Dollar P. Rethinking imageNet pretraining. Proceedings of the IEEE International Conference on Computer Vision; 2019 Nov 27 Oct.-2; Seoul, South Korea [Internet]. New York: IEEE; 2019 [cited 2021 May 7]. p. 4917-4926. Available from: https://ieeexplore. ieee.org/document/9010930. http://dx.doi.org/10.1109/ ICCV.2019.00502.

Hoiem D, Chodpathumwan Y, Dai Q. Diagnosing error in object detectors. 2012 [cited 2021 May 3]. In: University of Illinois Urbana-Champaign. The Grainger College of Engineering. Derek Hoiem [Internet]. Urbana, IL: Derek Hoiem; c2021. Available from: https://dhoiem.web.engr. illinois.edu/publications/eccv2012_detanalysis_derek.pdf. http://dx.doi.org/10.1007/978-3-642-33712-3_25.

Hothorn T, Müller J, Held L, Möst L, Mysterud A. Temporal patterns of deer-vehicle collisions consistent with deer activity pattern and density increase but not general accident risk. Accid Anal Prev. 2015;81:143-52. http://dx.doi.org/10.1016/j. aap.2015.04.037.

Huang GB, Ramesh M, Berg T, Learned-Miller E. Labeled Faces in the Wild: a database for studying face recognition in unconstrained environments. Amherst: University of Massachusetts; 2007. Technical Report 07-49.

Huijser MP, Delborgo Abra F, Duffield JW. Cost justification and an example of cost-benefit analyses of mitigation measures aimed at reducing collisions with Capybara in São Paulo State, Brazil. In: Proceedings of the 2013 International Conference on Ecology and Transportation (ICOET 2013); 2013; Scottsdale Arizona. [place unknown]: ICOET; 2013.

Khosla A, Jayadevaprakash N, Yao B, Fei-Fei L. Novel dataset for fine-grained image categorization. In: Proceedings of the IEEE Conf. Comput. Vision and Pattern Recognition, 2011 [Internet]. Stanford: Standford University; 2020 [cited 2020 Sept 21]. Available from: http://vision.stanford.edu/ aditya86/ImageNetDogs/. Click on Dataset Reference / Primary / pdf.

Kušta T, Keken Z, Ježek M, Kůta Z. Effectiveness and costs of odor repellents in wildlife-vehicle collisions: a case study in Central Bohemia, Czech Republic. Transp Res Part D Transp Environ. 2015;38:1-5. http://dx.doi.org/10.1016/j. trd.2015.04.017. 
Lesmerises F, Dussault C, St-Laurent MH. Major roadwork impacts the space use behaviour of gray wolf. Landsc Urban Plan. 2013;112(1):18-25. http://dx.doi.org/10.1016/j. landurbplan.2012.12.011.

Lin TY, Maire M, Belongie S, Hays J, Perona P, Ramanan D, Dollár P, Zitnick CL. Microsoft COCO: common objects in context. In: Proceedings of the European Conference on Computer Vision; 2014; Zurich, Switzerland [Internet]. Switzerland: Springer; 2014 [cited 2021 May 3]. p. 740-55. (Lecture Notes in Computer Science; vol. 8693). Available from: https://link. springer.com/chapter/10.1007\%2F978-3-319-10602-1_48. https://doi.org/10.1007/978-3-319-10602-1_48.

Liu W, Anguelov D, Erhan D, Szegedy C, Reed S, Fu CY, Berg AC. SSD: single shot multibox detector. In: Proceedings of the European Conference on Computer Vision; 2016; Amsterdam, The Netherlands [Internet]. Switzerland: Springer; 2016 [cited 2021 May 3]. p. 21-37. (Lecture Notes in Computer Science; vol. 9905). Available from: https://link. springer.com/chapter/10.1007\%2F978-3-319-46448-0_2. https://doi.org/10.1007/978-3-319-46448-0_2.

Munappy A, Bosch J, Olsson HH, Arpteg A, Brinne B. Data management challenges for deep learning. In: Proceedings of the 45th Euromicro Conference on Software Engineering and Advanced Applications (SEAA); 2019 Aug 28-30; Kallithea, Greece [Internet]. New York: IEEE; 2019 [cited 2021 May 3]. p. 140-147. Available from: https://ieeexplore. ieee.org/document/8906736. http://dx.doi.org/10.1109/ SEAA.2019.00030.

Oishi Y, Oguma H, Tamura A, Nakamura R, Matsunaga T. Animal detection using thermal images and its required observation conditions. Remote Sens. 2018;10(7):1050. http://dx.doi.org/10.3390/rs10071050.

Olson DD, Bissonette JA, Cramer PC, Green AD, Davis ST, Jackson PJ, Coster DC. Monitoring wildlife-vehicle collisions in the Information Age: how smartphones can improve data collection. J Wildlife Rehabil. 2014;34(3):7-16. http://dx.doi.org/10.1371/journal.pone.0098613.

PASCAL VOC Project. The PASCAL Visual Object Classes Challenge 2012 [Internet]. [place unknown]: VOC 2012; 2012. 4 Detection task, 2012 May 21 [cited 2020 Feb 12]. Available from: http://host.robots.ox.ac.uk/pascal/VOC/voc2012/ htmldoc/devkit_doc.html\#SECTION00050000000000000000.

Pathak AR, Pandey M, Rautaray S, Pawar K. Assessment of object detection using deep convolutional neural networks. In: Subhash B, Vikrant B, Anjali AC, Anil SH, Suresh CS, editors.
Intelligent computing and information and communication [Internet]. Singapore: Springer Nature; 2018 [cited 2021 May3].p. 457-66. Available from: https://link.springer.com/ chapter/10.1007\%2F978-981-10-7245-1_45. http://dx.doi. org/10.1007/978-981-10-7245-1_45.

Perissinotto M, Moura DJ, Cruz VF, Souza SRL, Lima KAO, Mendes AS. Conforto térmico de bovinos leiteiros confinados em clima subtropical e mediterrâneo pela análise de parâmetros fisiológicos utilizando a teoria dos conjuntos fuzzy. Cienc Rural. 2009;39(5):1492-8. http:// dx.doi.org/10.1590/S0103-84782009005000094.

Ponce J, Berg TL, Everingham M, Forsyth DA, Hebert M, Lazebnik S, Marszalek M, Schmid C, Russell BC, Torralba A, Williams CKI, Zhang J, Zisserman A. Dataset issues in object recognition. In: Ponce J, Hebert M, Schmid C, editors. Toward category-level object recognition [Internet]. Berlin: Springer; 2006 [cited 2021 May7]. p. 29-48. Available from: https://link.springer.com/chapter/10.1007\%2F11957959_2. http://dx.doi.org/10.1007/11957959_2.

Pynn TP, Pynn BR. Moose and other large animal wildlife vehicle collisions: implications for prevention and emergency care. J Emerg Nurs. 2004;30(6):542-7. http://dx.doi. org/10.1016/j.jen.2004.07.084.

Rangda MB, Hanchate DB. Animal detection using histogram oriented gradient. Int J Recent and Innov Trends Comput Commun. 2014;2(2):178-83.

Rawat W, Wang Z. Deep convolutional neural networks for image classification: a comprehensive review. Neural Comput. 2017;29(9):2352-449. http://www.mitpressjournals.org/ doi/abs/10.1162/neco_a_00990.http://dx.doi.org/10.1162/ neco_a_00990. PMid:28599112.

Rodríguez-Morales B, Díaz-Varela ER, Marey-Pérez MF. Spatiotemporal analysis of vehicle collisions involving wild boar and roe deer in NW Spain. Accid Anal Prev. 2013;60:121-33. http://dx.doi.org/10.1016/j.aap.2013.07.032.

Russakovsky O, Deng J, Su H, Krause J, Satheesh S, Ma S, Huang Z, Karpathy A, Khosla A, Bernstein M, Berg $A C$, Fei-Fei L. ImageNet large scale visual recognition challenge. Int J Comput Vis. 2015;115(3):211-52. http:// dx.doi.org/10.1007/s11263-015-0816-y.

Sharma SU, Shah DJ. A practical animal detection and collision avoidance system using computer vision technique. IEEE Access. 2017;5:347-58. http://dx.doi.org/10.1109/ ACCESS.2016.2642981. 
Sullivan JM. Trends and characteristics of animal-vehicle collisions in the United States. J Safety Res. 2011;42(1):916. http://dx.doi.org/10.1016/j.jsr.2010.11.002.

Tavares DM, Coelho IB, Cardoso PH, Lima RPO, Stein PP. Cenário dos acidentes por atropelamento de fauna nas rodovias federais brasileiras e a mortalidade dos humanos envolvidos. In: Anais do XXXI Congresso de Pesquisa e Ensino em Transportes [Internet]. [place unknown]: ANPET; 2017 [cited 2020 Feb 12]. Available from: http://www.anpet. org.br/tempsite/xxxianpet/index.php/artigos-novo.

Tissier ML, Jumeau J, Croguennec C, Petit O, Habold C, Handrich Y. An anti-predation device to facilitate and secure the crossing of small mammals in motorway wildlife underpasses. (I) Lab tests of basic design features. Ecol Eng. 2016;95:738-42. http://dx.doi.org/10.1016/j. ecoleng.2016.07.012.

Tzutalin D. LabelImg [2015] - [cited 2020 Feb 12]. In: GitHub [Internet]. [place unknown]; c2020. Available from: https://github.com/tzutalin/labelImg.

Wadey J, Beyer HL, Saaban S, Othman N, Leimgruber P, Campos-Arceiz A. Why did the elephant cross the road? The complex response of wild elephants to a major road in Peninsular Malaysia. Biol Conserv. 2018;218:91-8. http:// dx.doi.org/10.1016/j.biocon.2017.11.036.
Weiss K, Khoshgoftaar TM, Wang D. A survey of transfer learning. J Big Data. 2016;3(1):9. http://dx.doi.org/10.1186/ s40537-016-0043-6.

Ye T, Wang B, Song P, Li J. Automatic railway traffic object detection system using feature fusion refine neural network under shunting mode. Sensors. 2018;18(6):1916. http:// dx.doi.org/10.3390/s18061916.

Yu M, Yang P, Wei S. Railway obstacle detection algorithm using neural network. AIP Conf Proc. 2018;1967(May):040017. http://dx.doi.org/10.1063/1.5039091.

Zhou D. Thermal image-based deer detection to reduce accidents due to deer-vehicle collisions [Internet]. Minneapolis: Intelligent Transportation Systems Institute, Center for Transportation Studies, University of Minnesota; 2013 Jan[cited 2020 Feb 12]. Available from: https://conservancy. umn.edu/handle/11299/144870.

Zhuang F, Qi Z, Duan K, Xi D, Zhu Y, Zhu H, Xiong H, He Q. A comprehensive survey on transfer learning. 2019 Nov 7 [cited 2020 Feb 12]. In: arXiv [Internet]. New York: Cornell University; 2020. Available from: http://arxiv.org/ abs/1911.02685.

Financial Support: The Donkey Sanctuary through FUMVET - Fundação Medicina Veterinária and Intervias (Grupo Arteris). 